\title{
Tesis españolas sobre Historia de América Latina. Análisis general y recopilación bibliográfica de tesis sobre Historia Contemporánea (1976/77-1996/97)
}

\author{
Luis Rodríguez Yunta \\ Centro de Información, Documentación Científica \\ (CINDOC)-CSIC. Madrid
}

\begin{abstract}
El objetivo de este trabajo es contribuir a la necesaria difusión de la investigación histórica latinoamericanista desarrollada en España, a partir de la recopilación y análisis de las tesis leídas en universidades españolas relativas a la Historia de América Latina. A partir de esta recopilación, se realiza un breve análisis de las tendencias de la investigación científica desarrollada en este campo en nuestro país. Se incluyen 262 referencias de tesis de Historia Contemporánea, clasificadas por aspectos temáticos.
\end{abstract}

\section{Introducción}

Las tesis doctorales constituyen una fuente bibliográfica que merece un tratamiento específico por parte de la documentación científica. Cada una de ellas es el fruto de un trabajo de investigación de larga duración, desarrollado bajo una dirección desempeñada por un investigador de competencia contrastada. Se trata de investigaciones exhaustivas que exigen un tratamiento novedoso y conllevan muchas horas de trabajo. El proceso de evaluación por parte de un tribunal nos asegura la calidad de estos trabajos. Todo este esfuerzo no merece que esta documentación pueda caer en el olvido.

Sin embargo, en España la difusión de las tesis es en gran parte una tarea pendiente, ya que no existe un depósito central que garantice la accesibilidad de estos documentos y facilite su reproducción o consulta. Para la localización de tesis es necesario acudir a cada una de las respectivas universidades en las que encontraremos normas muy diferentes. La base de datos Teseo del Consejo de Universidades reúne un gran número de referencias de tesis españolas, pero su consulta es de poca utilidad para los trabajos de búsqueda bibliográfica al no garantizar el acceso a los documentos originales, cuya responsabilidad pertenece a cada una de las universidades. 
El objetivo de este trabajo es doble. Por un lado, pretende contribuir a la necesaria difusión de esta documentación científica, a través de la recopilación y clasificación de las tesis relativas a la Historia de América Latina ${ }^{1}$ leídas desde el curso 1976-1977, incluyendo en este artículo solamente las referencias relativas a la Edad Contemporánea por razones de espacio. En segundo lugar, esta recopilación nos va a permitir analizar las tendencias de la investigación histórica latinoamericanista desarrollada en España durante estos últimos veinte años. Debido a las características propias de estos documentos, podemos considerar la evolución de la lectura de tesis latinoamericanistas en las universidades españolas como uno de los indicadores más relevantes para el análisis del estado de la investigación científica desarrollada en este campo en nuestro país. La validez de este indicador debe matizarse: parte de los doctorandos son becarios latinoamericanos que realizaron su tesis en España. Pero este hecho no las convierte en investigaciones ajenas al latinoamericanismo español, sino que lo enriquecen notablemente. Este estudio no pretende, por tanto, realizar un ambicioso estado de la cuestión de este campo de investigación, sino tan sólo contribuir a su análisis parcial, siguiendo una línea de estudios bibliométricos ya iniciada por otros autores ${ }^{2}$.

Podremos comparar las tendencias del latinoamericanismo español con las de algunos otros países europeos, a partir de los datos reunidos en la base de datos de Tesis Europeas sobre América Latina, en cuya elaboración hemos contribuido aportando las referencias de la parte española. Esta base de datos se configuró a partir de un repertorio impreso elaborado por la Red Europea de Documentación e Información sobre América Latina (REDIAL) en $1992^{3}$. Actualmente reúne casi cinco mil referencias.

El análisis desarrollado en este artículo se centra en las tesis relativas a la Historia de América Latina. Este sector es especialmente predominante en el americanismo español. Su importancia es también relevante dentro

1 Para la justificación del uso del término América Latina merece la pena consultar: Quijada, Mónica: "Sobre el origen y difusión del nombre 'América Latina' (o una variación heterodoxa en torno al tema de la construcción social de la verdad)", Revista de Indias, vol. LVIII, n. ${ }^{2}$ 214, Madrid, 1998, págs. 595-615.

2 Duport, Claudie: "Tesis doctorales europeas sobre América Latina, 1980-1989. Datos bibliométricos y algunos comentarios", REDIAL. Revista Europea de Información y Documentación sobre América Latina, n. ${ }^{\circ}$ 2, Paris, 1993, págs. 21-28. Vidal, Laurent: "Cuestión urbana y América Latina. Estudios de doctorado en Francia (1980-1990)", REDIAL. Revista Europea de Información y Documentación sobre América Latina, n. ${ }^{\circ}$ 1, Paris, 1992, págs.51-82.

3 Román Román, Adelaida (coord.): Repertorio de tesis europeas sobre América Latina 1980-1990, Madrid, 1992. 
del conjunto de la Historiografía española. En un país tradicionalmente ocupado en reflexionar sobre su propia Historia, con muy pocos especialistas en otras zonas geográficas, la Historia de América supone la única excepción que reúne a un alto número de investigadores.

\section{Fuentes utilizadas}

La mayoría de las referencias están extraídas de la base de datos TESEO del Consejo de Universidades (http://www.mec.es/teseo/). La localización de las mismas no es una tarea sencilla, al carecer de descriptores precisos para este tipo de búsqueda. Sin embargo, esta base de datos no reúne la totalidad de las tesis españolas, por lo que se ha procurado enriquecer esta selección, en la medida de lo posible, con otras fuentes complementarias: los repertorios impresos del propio Consejo de Universidades, la base de datos Tesis Doctorales de la Universidad Complutense de Madrid (http://www.ucm.es/bucm/busqueda_tesis.htm) y el Directorio de Americanistas Españoles. ${ }^{4}$

Se han analizado las tesis encontradas sobre cualquier aspecto histórico relativo a América Latina, exceptuando la Historia de la Literatura, pero incluyendo disciplinas como la Historia del Arte, la Arqueología, la Historia de la Lengua, la Historia Económica o la Historia de las Relaciones Internacionales. Por razones de espacio, se incluyen en este artículo exclusivamente las referencias de tesis relativas a la Historia Contemporánea de América, aunque el análisis que a continuación se desarrolla se refiere a la totalidad de las tesis localizadas. De un total de 1449 registros de tesis españolas existentes en la base de datos "Tesis Europeas sobre América Latina", 633 pueden considerarse como investigaciones históricas, de las cuáles se incluyen en este artículo las referencias de 262 tesis de Historia Contemporánea.

A pesar de las ausencias que sin duda existirán en esta recopilación, espero que resulte de interés para los lectores de esta revista. Este recurso de información continuará actualizándose en la base de datos REDIAL-TESIS, a la cual puede accederse a través de internet, bien desde la página web del CINDOC (http://www.cindoc.csic.es) o desde REDIAL (http://www.eurosur.org/REDIAL/redial.html), y en la cual podrá consul-

4 Román Román, Adelaida: Directorio de Americanistas Españoles, Madrid, 1998. 
tarse el conjunto completo de referencias bibliográficas de tesis sobre Historia de América que no puede recogerse en el formato de un artículo de revista.

\section{Análisis general de las tesis españolas de Historia de América}

Los datos que aquí se presentan están condicionados por variables de difícil control: las lagunas existentes en las fuentes consultadas, la evolución de la presencia de doctorandos latinoamericanos en nuestro país, el desarrollo de las becas antes y después del Quinto Centenario, etc. Por consiguiente, hay que matizar que dichos datos no deben tomarse más que como meros indicadores relativos de las tendencias existentes en este sector de la investigación.

En el siguiente gráfico se presenta la evolución cuantitativa del número de tesis leídas en las universidades españolas relativas a Latinoamérica en el conjunto de las Ciencias Sociales y Humanas en comparación con las de Historia de América Latina. Puede apreciarse un claro crecimiento a partir del curso 1986-1987, alcanzando desde este momento un valor

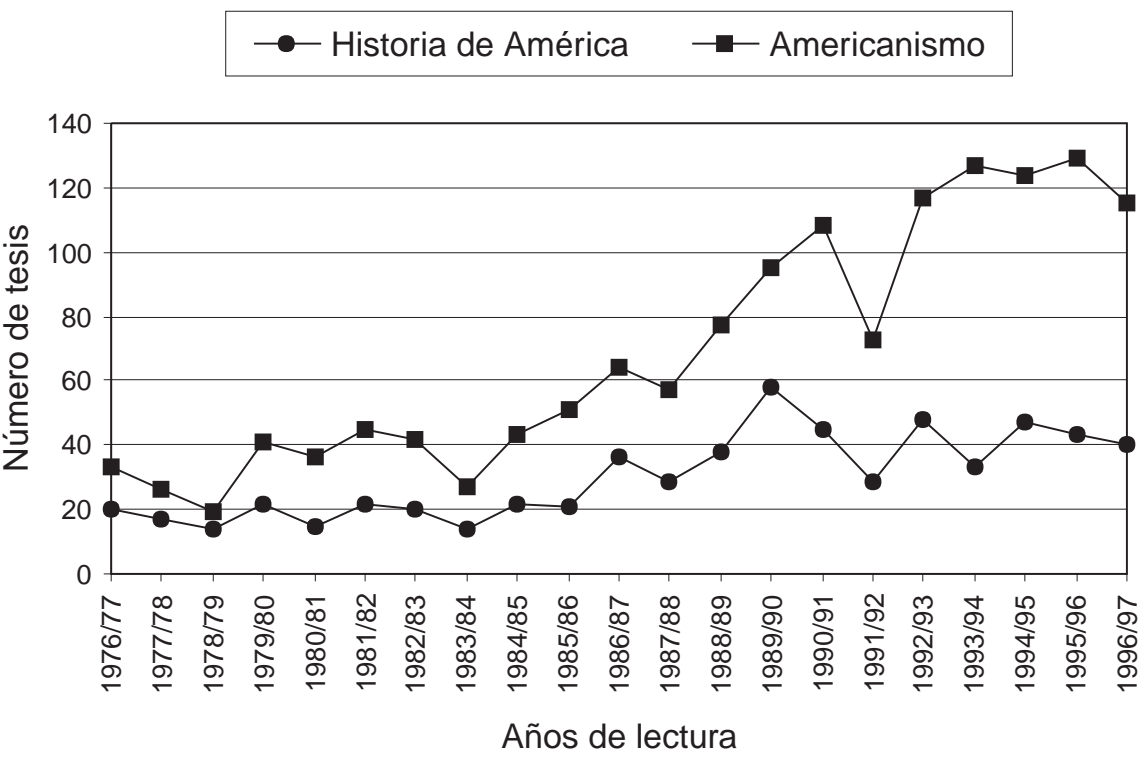


medio de 40 tesis anuales de Historia de América, con variaciones entre las 30 y las casi 60 tesis (curso 1989-1990). Sin embargo, en el conjunto general de las tesis americanistas españolas se observa un mayor crecimiento a partir del curso 1992-1993 que no se ve correspondido en el ámbito concreto de la Historia.

Por tanto, puede decirse que el peso específico de este área temática tiende a reducirse dentro del americanismo desarrollado a través de las tesis españolas, evolucionando desde el 74\% en el curso 1978-1979 hasta sólo un 26\% en 1993-1994. Aunque las tesis de Historia de América mantienen unas cifras anuales bastante estables, su crecimiento es menor que el de otras disciplinas dentro del americanismo.

Este peso relativo de la Historia dentro del americanismo español queda palpable en otras fuentes consultadas: está representado por el $56 \%$ de los investigadores que figuran en el Directorio de Americanistas Españoles ${ }^{5}$ y por el $35 \%$ de las referencias incluidas en la base de datos ISOC-ALAT ${ }^{6}$. Su peso relativo, no obstante, continúa siendo superior al que representa la Historia dentro del americanismo desarrollado en otros países europeos, como se observa en los siguientes datos extraídos de la base de datos de REDIAL "Tesis europeas sobre América Latina":

\begin{tabular}{lccr} 
Países & Tesis americanistas & Tesis Historia de América & Porcentaje \\
\hline España (1976-97) & 1449 & 633 & $44 \%$ \\
R. Unido (1980-97) & 1424 & 126 & $9 \%$ \\
Francia (1980-96) & 978 & 187 & $19 \%$ \\
Alemania (1980-1994) & 356 & 25 & $7 \%$ \\
Austria (1980-96) & 196 & 21 & $11 \%$ \\
Bélgica (1980-90) & 153 & 9 & $6 \%$ \\
Países Bajos (1979-96) & 106 & 7 & $7 \%$ \\
Suecia (1990-1997) & 45 & 3 & $7 \%$
\end{tabular}

A continuación se desglosan las Universidades y Facultades donde han sido leídas las tesis localizadas. No debe interpretarse esta tabla como un ranking sino como una información relevante sobre la localización

5 Román: Directorio...

6 ALAT es un subfichero de la base de datos ISOC, gestionada por el CINDOC, que vacía la literatura científica española en Ciencias Sociales y Humanidades: artículos de revista, ponencias e informes publicados desde 1975. Está accesible desde las páginas web de dicho centro: www.cindoc.csic.es. 
geográfica de los centros de investigación. Destacan con claridad los dos centros tradicionales del americanismo español, la Universidad Complutense $(42 \%)$ y la Universidad de Sevilla (19\%), pero también debe destacarse el alto grado de dispersión de la investigación desarrollada en este campo, con 28 Universidades y 85 Facultades diferentes. No se puede analizar adecuadamente la distribución por departamentos ya que este dato sólo figura en el $43 \%$ de las referencias obtenidas.

Universidad Autónoma de Barcelona $\quad 8$

Fac. Ciencias Económicas y Empresariales 1

Fac. Filosofía i Lletres 6

Fac. Medicina 1

Universidad Autónoma de Madrid 7

Fac. Ciencias Económicas y Empresariales 1

Fac. Filosofía y Letras 6

Universidad de Alcalá de Henares: 9

Fac. Ciencias Económicas y Empresariales 1

Fac. Filosofía y Letras 5

Fac. Medicina 3

Universidad de Alicante: $\quad 2$

Fac. Filosofía y Letras 2

Universidad de Barcelona 36

Fac. Ciencias Económicas y Empresariales 4

Fac. Filología 1

Fac. Filosofía y Ciencias de la Educación 2

Fac. Filosofía y Letras 3

Fac. Filosofía 1

Fac. Geografía i Historia 22

Fac. Pedagogía 3

Universidad de Cádiz $\quad 7$

Esc. Ingeniería Naval 1

Fac. Filosofía y Letras 4

Fac. Medicina 2

Universidad de Cantabria 2

Fac. Filosofía y Letras 2

Universidad de Complutense de Madrid 266

Fac. Bellas Artes 1

Fac. Biológicas $\quad 5$ 
Fac. Ciencias de la Información 7

Fac. Ciencias Económicas y Empresariales 5

Fac. Ciencias Políticas y Sociología 32

Fac. Derecho 9

Fac. Farmacia 2

Fac. Filología 11

Fac. Filosofía y Ciencias de la Educación $\quad 10$

Fac. Filosofía y Letras 19

Fac. Filosofía 2

Fac. Geografía e Historia 161

Fac. Medicina 2

Universidad de Córdoba 3

Esc. Tec. Sup. Ingenieros Agrónomos 1

Fac. Filosofía y Letras 2

Universidad de Deusto 2

Universidad de Extremadura 1

Fac. Medicina 1

Universidad de Granada $\quad 16$

Fac. Ciencias 1

Fac. Farmacia 2

Fac. Filosofía y Letras 13

Universidad de La Laguna $\quad 6$

Fac. Ciencias de la Información 1

Fac. Filosofía y Ciencias de la Educación 1

Fac. Geografía e Historia 4

Universidad de Málaga 1

Fac. Medicina 1

Universidad de Murcia $\quad 8$

Fac. Derecho 1

Fac. Letras $\quad 7$

Universidad de Navarra $\quad 38$

Fac. Arquitectura 1

Fac. Ciencias de la Información 2

Fac. Derecho 6

Fac. Derecho Canónico 1

Fac. Filosofía y Letras 9

Fac. Teología 19 
Universidad de Oviedo 5

Fac. Ciencias de la Educación 2

Fac. Geografía e Historia 3

Universidad de Salamanca $\quad 10$

Fac. Ciencias de la Educación 2

Fac. Derecho 1

Fac. Filosofía y Ciencias de la Educación 1

Fac. Filosofía y Letras 1

Fac. Geografía e Historia 5

Universidad de Santiago de Compostela 2

Fac. Ciencias Económicas y Empresariales 1

Fac. Filosofía y Ciencias de la Educación 1

Universidad de Sevilla 120

Fac. Ciencias Económicas y Empresariales 1

Fac. Derecho 1

Fac. Filología 2

Fac. Filosofía y Ciencias de la Educación 1

Fac. Filosofía y Letras $\quad 15$

Fac. Geografía e Historia $\quad 97$

Fac. Medicina 3

Universidad de Valencia $\quad 10$

Fac. Filología 1

Fac. Geografía e Historia 8

Fac. Medicina y Odontología 1

Universidad de Valladolid $\quad 42$

Fac. Arquitectura 1

Fac. Derecho 1

Fac. Filosofía y Letras 39

Fac. Medicina y Cirugía 1

Universidad de Zaragoza $\quad 7$

Fac. Derecho 1

Fac. Filosofía y Letras 6

Universidad del País Vasco 4

Fac. Filología, Geografía e Historia 4

Universidad Nacional de Educación a Distancia $\quad 8$

Fac. Filosofía 2

Fac. Filosofía y Ciencias de la Educación 2

Fac. Geografía e Historia 4 


$\begin{array}{cc}\text { Universidad Politécnica de Cataluña } & 5 \\ \text { Esc. Tec. Sup. Arquitectura } & 5 \\ \text { Universidad Politécnica de Madrid } & 6 \\ \text { Esc. Ingenieros de Montes } & 1 \\ \text { Esc. Tec. Sup. Arquitectura } & 5 \\ \text { Universidad Politécnica de Valencia } & 2 \\ \text { Fac. Bellas Artes } & 2\end{array}$

El predominio de la Universidad Complutense de Madrid y la Universidad de Sevilla tiende a disminuir con el tiempo al surgir nuevas universidades que contribuyen a una mayor dispersión del latinoamericanismo. En el período 1976-1985 reúnen entre las dos el 67\% de las referencias localizadas, mientras que para la década siguiente suponen sólo el 54\%. La Universidad Complutense es especialmente preponderante en algunas áreas temáticas dentro del latinoamericanismo, en concreto se sitúa por encima de su valor medio (42\%) dentro de las siguientes disciplinas: Arqueología (86\%), Historia precolombina (75\%), Historia Contemporánea (57\%), Historia política (60\%) e Historia del Derecho (52\%). Por su parte la Universidad de Sevilla destaca por encima de su valor medio (19\%) en: Historia colonial (35\%), Historia militar (46\%), Historia social $(35 \%)$ e Historia económica (33\%).

Las Facultades de Geografía e Historia y de Filosofía y Letras representan lógicamente el monto mayor de las tesis recopiladas (71\%), aunque es un hecho destacable que el 29\% procedan de otras Facultades y Escuelas ajenas a la Historia. Igualmente, destaca el elevado número de investigadores diferentes (221) que figuran como directores de las tesis seleccionadas. Como profesores que han dirigido mayor número de tesis de Historia de América en estos veinte años, según los datos que figuran en esta recopilación, hay que destacar a Mario Hernández Sánchez-Barba (18), Paulino Castañeda Delgado (15), Manuel Ballesteros Gaibrois (14), Luis Navarro García (12), Demetrio Ramos Pérez (11) e Ismael Sánchez Bella (10). Todas estas cifras podrían ser mayores, ya que tan sólo se ha localizado este dato en el $65 \%$ de las referencias.

En cuanto a los países latinoamericanos más estudiados, destaca sobre todo México, con 134 tesis, lo que supone un 21\%. Le siguen a mucha distancia Argentina (56), Perú (54), Cuba (49), Puerto Rico (44), Venezuela (41), Chile (38), Colombia (33) y Brasil (21). 


\section{Relación de las tesis españolas de Historia Contemporánea de América Latina}

Se han clasificado las 262 tesis localizadas de Historia Contemporánea de América Latina en tres bloques: Movimientos de Independencia, Historia Colonial (Antillas en el siglo XIX) e Historia Contemporánea tras la Independencia. Estos dos últimos grupos se subdividen en subconjuntos temáticos por aspectos como la Historia Política, Relaciones Internacionales, Historia Económica, Historia Social, Historia de la Religión, Historia Cultural, Historia de la Ciencia, etc.

\section{Movimientos de independencia (1810-1824)}

1. BerRuezo LeÓn, María Teresa: Inglaterra 1800-1830: liberales españoles e hispanoamericanos ante la independencia de América. Univ. Complutense de Madrid, Fac. Geografía e Historia, 1987-1988.

2. Chust Calero, Manuel: La cuestión americana en las Cortes de Cádiz (1808-1814). Sebastiá Domingo, Enric (Dir.). Univ. Valencia, Fac. Geografía e Historia, Dep. Historia Contemporánea, 1992-1993.

3. Colomer Pellicer, Francisca: Baltasar Hidalgo de Cisneros, último virrey del virreinato del Río de la Plata. Una biografía histórica. Andreo García, Juan (Dir.). Univ. Murcia, Fac. Letras, Dep. Historia Moderna, Contemporánea y de América, 1996-1997.

4. FERnÁndez PASCUA, Delfina: La resistencia española en Venezuela después de Carabobo y el respaldo de Puerto Rico. Univ. Valladolid, Fac. Filosofía y Letras, 1988-1989.

5. GÁlvez Montero, José Francisco: Las bases del estado peruano y el proceso de independencia (1821-1828). Pérez-Prendes y Muñoz de Arraco, José Manuel (Dir.). Univ. Complutense de Madrid, Fac. Geografía e Historia, Dep. Historia Contemporánea, 1996-1997.

6. Gómez BARBozA, Paulina Rosa: La mujer de Gran Colombia ante el proceso político emancipador. Un estudio de género y de mentalidades. Stolcke, Verena (Dir.). Univ. Complutense de Madrid, Fac. Geografía e Historia, Dep. Historia Contemporánea, 1995-1996.

7. Herrera VAldés, Willy Francisco: Las sociedades secretas y el proceso de independencia del cono suramericano: O'Higgins, San Martín y la logia Lautaro. Univ. Complutense de Madrid, Fac. Geografía e Historia, 1984-1985.

8. MARTínez RiAZA, Ascensión: Conciencia nacional e ideología política en la sociedad peruana, 1811-1824: La prensa doctrinal en la independencia. Hernández Sánchez-Barba, Mario (Dir.). Univ. Complutense de Madrid, Fac. Geografía e Historia, Dep. Historia de América, 1982-1983. 
TESIS ESPAÑOLAS SOBRE HISTORIA DE AMÉRICA LATINA (1976/77-1996/97)

9. Olmos SÁnchez, Isabel: La sociedad mexicana en vísperas de la Independencia. Lucena Salmoral, Manuel (Dir.). Univ. Murcia, Fac. Letras, Dep. Historia de América, 1984-1985.

10. Pérez Turrado, Gaspar: La Marina española en la independencia de Costa Firme. Morales Padrón, Francisco (Dir.). Univ. Sevilla, Fac. Geografía e Historia, 1982-1983.

11. Pumar Martínez, Carmen: La crisis del virreinato en el Nuevo Reino de Granada (1803-1810). Univ. Alcalá de Henares, Fac. Filosofía y Letras, 1986-1987.

12. Rengifo CARrillo, Diana: La unidad regional Caracas-La Guaira-Valles ante el impacto social y económico de la Revolución de Independencia, 1775-1825. Hernández Sánchez-Barba, Mario (Dir.). Univ. Complutense de Madrid, Fac. Geografía e Historia, 1978-1979.

13. Rodríguez León, Mario A.: El obispo Juan Alejo de Arizmendi ante el proceso revolucionario y la emancipación de América Latina y el Caribe. Ramos Pérez, Demetrio (Dir.). Univ. Valladolid, Fac. Filosofía y Letras, Dep. Historia Moderna, Contemporánea y de América, 1990-1991.

\section{Historia Colonial-Antillas en el siglo XIX}

a) Gobierno y administración colonial

14. Basualdo Miranda, Hugo Roberto: Claudio Martínez de Pinillos y la superintendencia general delegada de la Real Hacienda de la isla de Cuba (1825-1851). González Loscertales, Vicente (Dir.). Univ. Complutense de Madrid, Fac. Geografía e Historia, 1985-1986.

15. Burgos Malave, Eda Milagros: La carta autonómica de 1897 en Puerto Rico. Sánchez Bella, Ismael (Dir.). Univ. Navarra, Fac. Filosofía y Letras, 1981-1982.

16. Cayuela Fernández, José Gregorio: Estado, ejército y sociedad en las relaciones coloniales españolas. Cuba: 1854-1859. Univ. Complutense de Madrid, Fac. Geografía e Historia, Dep. Historia Contemporánea, 1989-1990.

17. Diego García, Emilio de: Puerto Rico bajo la administración española durante la primera mitad del siglo XIX. Palacio Atard, Vicente (Dir.). Univ. Complutense de Madrid, Fac. Geografía e Historia, Dep. Historia Contemporánea, 1982-1983.

18. LABARCA Bonnet, René Luis: Elecciones y partidos políticos de Cuba: 1810-1898. Univ. Complutense de Madrid, Fac. Ciencias Políticas y Sociología, 1976-1977. 
19. LagunA OchOA, Fernando: Las ideas hispanoamericanistas de Rafael María de Labra. (Ultramar y sus problemas durante el siglo XIX). Univ. Complutense de Madrid, Fac. Geografía e Historia, Dep. Historia de América, 1990-1991.

20. NAVArRo García, Jesús Raúl: Control y conflictos en Puerto Rico: la política española tras la independencia continental, 1824-1837. Univ. Sevilla, Fac. Geografía e Historia, Dep. Historia de América, 1987-1988.

21. RoldÁn De Montaud, Inés: La Unión Constitucional y la política colonial de España en Cuba (1868-1898). Univ. Complutense de Madrid, Fac. Geografía e Historia, Dep. Historia Contemporánea, 1989-1990.

22. Saiz PAStor, Candelaria: Liberales y esclavistas. El dominio colonial español en Cuba (1833-1868). Pérez Garzón, Juan Sisino (Dir.). Univ. Alicante, Fac. Filosofía y Letras, Dep. Historia Contemporánea, 1989-1990.

23. SÁNCHEZ ANDRÉs, Agustín: La política colonial española, 1810-1898: administración central y estatuto antillano. Pereira Castañares, Juan Carlos (Dir.). Univ. Complutense de Madrid, Fac. Geografía e Historia, Dep. Historia Contemporánea, 1995-1996.

24. VÁzQuez Sotillo, Nelly: La política gubernativa de orden y seguridad pública en Puerto Rico (1835-1865). Mijares Pérez, Lucio (Dir.). Univ. Valladolid, Fac. Filosofía y Letras, Dep. Historia Moderna, Contemporánea y de América, 1994-1995.

Véase además: 30, 35, 49 y 94.

b) Relaciones internacionales

25. Palomino Salguero, Francisca: Consulados extranjeros en Puerto Rico durante el siglo XIX. Cabrero Fernández, Leoncio (Dir.). Univ. Complutense de Madrid, Fac. Geografía e Historia, 1995-1996.

26. Rodríguez LóPEZ, Carlos: La geoestrategia del Caribe en el siglo XIX: la isla de Vieques. Univ. Complutense de Madrid, Fac. Geografía e Historia, Dep. Historia de América, 1990-1991.

27. Seviliano Castillo, Rosa: Cuba en el área de intereses internacionales atlánticos: 1819-1861. Univ. Complutense de Madrid, Fac. Geografía e Historia, Dep. Historia de América, 1986-1987.

Véase además: 28.

c) Guerras de independencia y crisis del 98

28. Companys Monclus, Julia: La diplomacia norteamericana en torno al 98. Univ. Barcelona, Fac. Filosofía y Letras, Dep. Geografía e Historia, 1986-1987. 
29. Delgado Pasapera, Germán: Los movimientos independentistas de Puerto Rico en la segunda mitad del siglo XIX. Hernández Sánchez-Barba, Mario (Dir.). Univ. Complutense de Madrid, Fac. Geografía e Historia, Dep. Historia de América, 1981-1982.

30. Domingo Acebrón, M. Dolores: Proyección social y política de la guerra de los Diez Años: Cuba (1868-1878). Univ. Autónoma de Madrid, Fac. Filosofía y Letras, 1987-1988.

31. GonzÁlez Diao de Oria, Alicia: La emancipación cubana a través de las fuentes literarias. Univ. Valladolid, Fac. Filosofía y Letras, 1986-1987.

32. MAnso Platero, Francisco: La sanidad militar en la guerra de Cuba durante la I República. Zaragoza Rubira, Juan Ramón (Dir.). Univ. Sevilla, Fac. Medicina, 1980-1981.

33. MARTíneZ DE LAS Heras, Agustín: La crisis cubana en el arranque del sexenio democrático. Univ. Complutense de Madrid, Fac. Ciencias de la Información, 1984-1985.

34. Ojeda Reyes, Félix: Ramón Emeterio Betances: exilio y libertad. Mijares Pérez, Lucio (Dir.). Univ. Valladolid, Fac. Filosofía y Letras, Dep. Historia Moderna, Contemporánea y de América, 1994-1995.

35. Prada Velasco, María del Carmen: La Guerra de Yara (1868-1878). Aspectos políticos, sociales y económicos. Jover Zamora, José María (Dir.). Univ. Complutense de Madrid, Fac. Filosofía y Letras, 1977-1978.

d) Historia Económica

36. Calavera Vaya, Ana María: Relaciones bancarias hispano-cubanas. La Habana, plaza y mercado, 1829-1868. Hernández Ruigómez, Almudena (Dir.). Univ. Complutense de Madrid, Fac. Geografía e Historia, Dep. Historia de América I, 1992-1993.

37. Camuñas Madera, Ricardo: La conquista del área oeste de Puerto Rico por el capitalismo en el siglo XIX a través de la evolución de las grandes familias. Univ. Valladolid, Fac. Filosofía y Letras, 1987-1988.

38. Chover Serrano, J. Enrique: Análisis del tráfico marítimo entre Cádiz y las Antillas españolas (1891-1904). Piniella Corbacho, Francisco (Dir.). Univ. Cádiz, Esc. Ingeniería Naval, Dep. Ciencias y Técnicas de la Navegación, Máquinas y Motores, 1996-1997.

39. DíAz Hernández, Luis: Una hacienda de café en Puerto Rico: Castañer, 1868-1914. Sánchez Bella, Ismael (Dir.). Univ. Navarra, Fac. Filosofía y Letras, 1980-1981.

40. García-Montón García-Baquero, María Isabel: El sistema de correos interiores y el desarrollo regional en Cuba, 1754-1868. Univ. Complutense de Madrid, Fac. Geografía e Historia, 1989-1990. 
41. Hernández Ruigómez, Almudena: La desamortización eclesiástica de Puerto Rico en el siglo XIX. Pérez Cuesta, Alberto (Dir.). Univ. Complutense de Madrid, Fac. Geografía e Historia, 1985-1986.

42. Marte Roberto, Mario: Cuba y la República Dominicana: formas económicas en transición en el Caribe en el siglo XIX. Univ. Complutense de Madrid, Fac. Geografía e Historia, Dep. Historia de América, 1988-1989.

43. Mattei Rodríguez, Lucas: La Real Sociedad Económica de Amigos del País de Puerto Rico, desde sus orígenes hasta el final del Trienio Constitucional, verdadero registro de la mentalidad puertorriqueña. Ramos Pérez, Demetrio (Dir.). Univ. Valladolid, Fac. Filosofía y Letras, Dep. Historia Moderna, Contemporánea y de América, 1996-1997.

44. Piniella Corbacho, Francisco: La Empresa Mercantil de Correos Marítimos (1827-1851) - Aproximación a los usos náuticos. Pérez Murillo, M. Dolores (Dir.). Univ. Cádiz, Fac. Filosofía y Letras, Dep. Historia Moderna, Contemporánea, Arte y América, 1993-1994.

45. Robles Martínez, Wilma: Origen y desarrollo histórico de las cajas de ahorro en Puerto Rico. Univ. Complutense de Madrid, Fac. Geografía e Historia, Dep. Historia de América, 1989-1990.

Véase además: 14, 25 y 35.

e) Historia Social. Demografia

46. Castellano Gil, José Manuel: La masonería de obediencia española en Cuba durante el siglo XIX. Paz Sánchez, Manuel de (Dir.). Univ. La Laguna, Fac. Geografía e Historia, Dep. Historia, 1992-1993.

47. Pérez Murillo, M. Dolores: Aspectos demográficos y sociales de la isla de Cuba en el segundo cuarto de siglo XIX. Navarro García, L. (Dir.). Univ. Sevilla, Fac. Geografía e Historia, 1986-1987.

48. PÉREZ Vega, Ivette: Las oleadas de inmigración sobre el sur de Puerto Rico: el caso de las sociedades mercantiles creadas en Ponce, 1816-1830. Univ. Valladolid, Fac. Filosofía y Letras, Dep. Historia de América, 1985-1986.

49. Rivera Rivera, Antonia: El papel del Estado español en la beneficencia del Puerto Rico del siglo XIX. Univ. Complutense de Madrid, Fac. Ciencias Políticas y Sociología, 1984-1985.

50. Rosario Rivera, Raquel: Efectos de las revoluciones en el poblamiento de Puerto Rico, 1789-1848. Univ. Valladolid, Fac. Filosofía y Letras, Sem. Historia de América, 1987-1988.

Véase además: 16,30 y 35. 


\section{f) Historia de la Religión}

51. Guerrero Cano, Magdalena: Santo Domingo en el período de anexión a España y Granada durante el Sexenio Revolucionario. Dos conflictos y un arzobispo: D. Bienvenido Monzón. Muñoz Pérez, José (Dir.). Univ. Granada, Fac. Filosofía y Letras, 1983-1984.

Véase además: 41.

g) Historia Cultural y de la Educación

52. Huerta Martínez, Angel: La enseñanza de las primeras letras en Cuba en el siglo XIX (1812-1868). Macías Domínguez, Isabelo (Dir.). Univ. Sevilla, Fac. Geografía e Historia, Dep. Historia de América, 1989-1990.

53. Madrigal de las CASAs, Dennis: La Real Academia de Buenas Letras de Puerto Rico. Univ. Valladolid, Fac. Filosofía y Letras, 1987-1988.

\section{h) Historia de la Ciencia}

54. GonZÁlez EsCRIG, José Luis: Estudios botánico-forestales españoles durante el siglo XIX. Fernández Pérez, Joaquín (Dir.). Univ. Complutense de Madrid, Fac. Biología, Dep. Biología Vegetal I, 1996-1997.

55. LóPEZ REVORA, Lourdes Teresa: Higiene y patología naval española de la segunda mitad del siglo XIX, en el departamento marítimo de Cádiz y sus relaciones con América. Univ. Cádiz, Fac. Medicina, Cat. Historia de la Medicina, 1988-1989.

Véase además: 44 y 234.

\section{Historia Contemporánea de los países}

latinoamericanos tras la independencia

a) Historia Política

56. Abnino ChicA, Antonio: Chile: cultura política y sociedad (1973-1988). Univ. Complutense de Madrid, Fac. Ciencias Políticas y Sociología, 1988-1989.

57. Álvarez Álvarez, Juan Rodrigo: Las relaciones entre partidos políticos, Iglesia, Fuerzas Armadas y gremios con el estado de Colombia de 1934 a 1962. Pérez-Prendes y Muñoz de Arraco, José Manuel (Dir.). Univ. Complu- 
tense de Madrid, Fac. Geografía e Historia, Dep. Historia de América, 1992-1993.

58. BARAHONA RIERA, Macarena: La lucha sufragista de la mujer en Costa Rica, 1890-1949. Univ. Complutense de Madrid, Fac. Ciencias Políticas y Sociología, Dep. Ciencia Política y de la Administración, 1989-1990.

59. BARrios FERRER, Gonzalo: Venezuela: cambio social y modernización política. Martínez Cuadrado, Miguel (Dir.). Univ. Complutense de Madrid, Fac. Ciencias Políticas y Sociología, Dep. Ciencia Política, 1978-1979.

60. BenAVENT LlaRio, Rafael: Evolución política y desarrollo económico de Puerto Rico. Morodo Leoncio, Raúl (Dir.). Univ. Complutense de Madrid, Fac. Ciencias Políticas y Sociología, Dep. Ciencia Política, 1982-1983.

61. Casaus Arzú, Marta Elena: La ideología de la clase dominante guatemalteca. Univ. Complutense de Madrid, Fac. Ciencias Políticas y Sociología, 1988-1989.

62. Castilla CRespi, José Luis: Análisis y evolución de los movimientos de liberación nacional en Iberoamérica: violencia y política, el caso peruano. Univ. Complutense de Madrid, Fac. Geografía e Historia, Dep. Historia de América, 1988-1989.

63. Chirinos Montalbetti, Ida María del Rocío: La constitución peruana de 1933. Sánchez Bella, Ismael (Dir.). Univ. Navarra, Fac. Filosofía y Letras, 1986-1987.

64. Contreras Solís, Fernando: Fundamentos político-sociales del estado liberal costarricense (1821-1940). Oya González, Jesús J. (Dir.). Univ. Complutense de Madrid, Fac. Ciencias Políticas y Sociología, Dep. Historia del Pensamiento y de los Movimientos Sociales y Políticos, 1992-1993.

65. DANes Rojas, Edgar: El Estado mexicano y la Iglesia Católica: evolución histórica y jurídica de sus relaciones. Navarro Valls, Rafael (Dir.). Univ. Complutense de Madrid, Fac. Derecho, Dep. Derecho Constitucional, 1996-1997.

66. FERNÁNDEZ APONTE, Irene: Los problemas del cambio de soberanía en Puerto Rico y la comprobación en la narrativa. Ramos Pérez, Demetrio (Dir.). Univ. Valladolid, Fac. Filosofía y Letras, Dep. Historia Moderna, Contemporánea y de América, 1990-1991.

67. FERnÁNDEZ FonTENOY, Carlos: Visión de la clase dirigente en el pensamiento político peruano 1900-1930. Univ. Complutense de Madrid, Fac. Ciencias Políticas y Sociología, 1989-1990.

68. Forero Gutiérrez, Alfonso: La prensa y el Estado liberal en Colombia, 1850-1880. Martínez Riaza, Ascensión (Dir.). Univ. Complutense de Madrid, Fac. Geografía e Historia, Dep. Historia de América I, 1994-1995.

69. FortunAtTI MolinA, Rodolfo: La transformación histórica del paradigma democrático en Chile: 1810-1964. Univ. Complutense de Madrid, Fac. Ciencias Políticas y Sociología, 1984-1985. 
70. Gallego MargalefF, Fernando José: Bolivia: génesis de una revolución. Las experiencias de reformismo militar tras la guerra del Chaco (1936-1939). Univ. Autónoma de Barcelona, Fac. Letras, Dep. Historia Moderna y Contemporánea, 1989-1990.

71. GARCíA AÑoveros, Jesús María: Estructura agraria y poder político en Guatemala: la reforma agraria de Arbenz. Hernández Sánchez-Barba, Mario (Dir.). Univ. Complutense de Madrid, Fac. Geografía e Historia, 1981-1982.

72. García Godoy, M. Teresa: El léxico del primer liberalismo español y mejicano (1810-1814). Estudio de lexicología sociopolítica. Mondéjar Cumpian, José (Dir.). Univ. Granada, Fac. Filosofía y Letras, Dep. Filología Española, 1994-1995.

73. GARCÍA JimÉNEZ, Encarnación: Supuestos económicos, sociales y políticos del triunfo y posterior fracaso de la vía chilena al socialismo. Univ. Autónoma de Madrid, Fac. Filosofía y Letras, 1988-1989.

74. Gugliano, Alfredo Alejandro: Democracia y modernización en Brasil, 1946-1961. Roitman Rosenmann, Marcos (Dir.). Univ. Complutense de Madrid, Fac. Ciencias Políticas y Sociología, Dep. Sociología I, 1996-1997.

75. HERnÁNDEZ NoRZAGARAY, Juan Ernesto: La liberalización política mexicana: legislación y procesos electorales en los estados de Baja California, Michoacán y Sinaloa (1977-1992). Paramio Rodrigo, Ludolfo (Dir.). Univ. Complutense de Madrid, Fac. Ciencias Políticas y Sociología, Dep. Ciencia Política y de la Administración I, 1995-1996.

76. Hidalgo Trenado, Manuel: El rendimiento del sistema venezolano de partidos, 1958-1988. Cotarelo García, Ramón (Dir.). Univ. Complutense de Madrid, Fac. Ciencias Políticas y Sociología, Dep. Ciencia Política y de la Administración II, 1994-1995.

77. IBÁÑEZ Rojo, Enrique: Los sindicatos en el gobierno. La crisis del movimiento obrero boliviano (1982-1985). Paramio Rodrigo, Ludolfo (Dir.). Univ. Complutense de Madrid, Fac. Ciencias Políticas y Sociología, Dep. Historia del Pensamiento I, 1996-1997.

78. IrurozQui Victoriano, Marta: Poder y élites en Bolivia, 1880-1920. Bases de dominio y mecanismos de reproducción. Martínez Riaza, Ascensión (Dir.). Univ. Complutense de Madrid, Fac. Geografía e Historia, Dep. Historia de América, 1991-1992.

79. LedESMA DE LAPEYRA, Viviana Alejandra: La transición democrática en Chile: Un ensayo de Historia política. Paz Sánchez, Manuel de (Dir.). Univ. La Laguna, Fac. Geografía e Historia, Dep. Historia, 1996-1997.

80. LóPEZ-Quecuty Puig, Concepción: Análisis de la revolución militar peruana, 1968-1973. Hernández Sánchez-Barba, Mario (Dir.). Univ. Complutense de Madrid, Fac. Filosofía y Letras, 1976-1977. 
81. Madrigal Fornos, Danilo: El desarrollo político de Nicaragua de 1821 hasta nuestros días. Recio Adrados, Juan Luis (Dir.). Univ. Complutense de Madrid, Fac. Ciencias Políticas y Sociología, Dep. Historia del Pensamiento y de los Movimientos Sociales y Políticos, 1995-1996.

82. MARTínez Rodríguez, Antonia: El sexenio alemanista. Modernización económica y proyecto político (1946-1952). Alcántara Sáez, Manuel (Dir.). Univ. Complutense de Madrid, Fac. Ciencias Políticas y Sociología, Dep. Ciencias Políticas y de la Administración, 1992-1993.

83. Mattei Filardi, Luis Antonio: El Consejo Ejecutivo de Puerto Rico, 1900-1909: una tutoría institucionalizada. Mijares Pérez, Lucio (Dir.). Univ. Valladolid, Fac. Filosofía y Letras, Dep. Historia Moderna, Contemporánea y de América, 1996-1997.

84. Medina Aravena, Juan Andrés: Frente Popular en España y Chile. Papel de las centrales sindicales: CTCH y UGT. Samaniego Boneu, Mercedes (Dir.). Univ. Salamanca, Fac. Geografía e Historia, Dep. Historia Medieval, Moderna y Contemporánea, 1992-1993.

85. MEDINA VIEDAS, Jorge: Concierto para la democracia (élites y clases en México). Paramio Rodrigo, Ludolfo (Dir.). Univ. Complutense de Madrid, Fac. Ciencias Políticas, Dep. Sociología I, 1993-1994.

86. Merino Huerta, Mauricio: El municipio en la formación del Estado nacional mexicano. Paramio Rodrigo, Ludolfo (Dir.). Univ. Complutense de Madrid, Fac. Ciencias Políticas y Sociología, Dep. Ciencia Política y de la Administración I, 1996-1997.

87. Montero Leal, Rulayda Rosario: La "época del caudillismo en Venezuela”. José Manuel Hernández y el proceso electoral de 1897. Univ. Barcelona, Fac. Geografía e Historia, 1989-1990.

88. PhelPs De CóRdova, Loretta: El Comisionado Residente de Puerto Rico: su inicio y el primer período de Félix Córdova Dávila. Ramos Pérez, Demetrio (Dir.). Univ. Valladolid, Fac. Filosofía y Letras, 1992-1993.

89. RiBes IbORRA, Vicente: La reforma y el Porfiriato en el estado de Aguascalientes. Ballesteros Gaibrois, Manuel (Dir.). Univ. Complutense de Madrid, Fac. Geografía e Historia, 1980-1981.

90. SANDERS, Karen: Nación y tradición: cinco discursos en torno a la nación peruana, 1885-1930. Alvira Domínguez, Rafael (Dir.). Univ. Navarra, Fac. Ciencias de la Información, Dep. Comunicación Pública, 1994-1995.

91. Sixirei Paredes, Carlos: El Brasil de Vargas, 1930-1945. Univ. Complutense de Madrid, Fac. Geografía e Historia, Dep. Historia de América, 1987-1988.

92. Teramo Pandolfi, M. Teresa: Juan Bautista Alberdi, periodista y defensor de la libertad de prensa. Acirón Royo, Ricardo (Dir.). Univ. La Laguna, Fac. Ciencias de la Información, 1992-1993.

Véase además: 131, 136, 141, 143, 175, 188, 192-193, 223-224 y 233. 


\section{b) Relaciones Internacionales}

93. Acosta RodríGuez, Carlos Manuel: Relaciones internacionales entre España y Panamá, 1914-1923. Univ. Complutense de Madrid, Fac. Geografía e Historia, 1989-1990.

94. Alañón Flox, Luis: La Marina y las expediciones en el Reinado de Isabel II. Torre Gómez, Hipólito de la (Dir.). Univ. Nal. Educación a Distancia, Fac. Geografía e Historia, Dep. Historia Contemporánea, 1992-1993.

95. Bermúdez Potes, Guillermo: Actitudes panameñas frente al Canal de Panamá, 1880-1980. Ballesteros Gaibrois, Manuel (Dir.). Univ. Complutense de Madrid, Fac. Geografía e Historia, 1983-1984.

96. Cal Martínez, M. Consuelo: España y Venezuela a mediados del siglo XIX: la reconciliación (1845-1851). Univ. Valladolid, Fac. Filosofía y Letras, Sem. Historia de América, 1987-1988.

97. Delgado Gómez-Escalonilla, Lorenzo: Acción cultural y política exterior. La configuración de la diplomacia cultural durante el régimen franquista (1936-1945). Espadas Burgos, Manuel (Dir.). Univ. Complutense de Madrid, Fac. Geografía e Historia, Dep. Historia Contemporánea, 1990-1991.

98. Delgado Larios, Almudena: España y la revolución mexicana (1910-1931). Univ. Complutense de Madrid, Fac. Geografía e Historia, Dep. Historia de América, 1989-1990.

99. ENRICH MARCET, Silvia: Historia diplomática entre España e Iberoamérica en el contexto de las relaciones internacionales (1955-1985). Univ. Complutense de Madrid, Fac. Geografía e Historia, 1986-1987.

100. Figallo Lascano, Beatriz: El protocolo Perón-Franco: relaciones hispano-argentinas, 1942-1952. Univ. Complutense de Madrid, Fac. Geografía e Historia, 1987-1988.

101. FigueroA EsQueR, Raúl Agustín: España ante la guerra entre México y los Estados Unidos, 1845-1848. Univ. Complutense de Madrid, Fac. Geografía e Historia, 1986-1987.

102. Flores TORRES, Oscar: Revolución mexicana y diplomacia española. Contrarrevolución y oligarquía hispana en México, 1909-1920. Gil Novales, Alberto (Dir.). Univ. Complutense de Madrid, Fac. Ciencias de la Información, Dep. Historia Contemporánea y Comunicación Social, 1990-1991.

103. Fonseca Herrera, Zaida María: Las relaciones diplomáticas entre Costa Rica y España en el Siglo XIX: aporte al conocimiento de las relaciones exteriores costarricenses. Univ. Complutense de Madrid, Fac. Geografía e Historia, Dep. Historia de América, 1986-1987.

104. Gimeno Gómez, Ana: La expedición del General Flores al Ecuador en 1846. Ramos Pérez, Demetrio (Dir.). Univ. Valladolid, Fac. Filosofía y Letras, Dep. Historia de América, 1983-1984. 
105. GonZÁlez de OleagA, Marisa Noemí: Las relaciones hispano-argentinas durante la segunda guerra mundial, 1939-1946. Identidad, ideología y crisis. Univ. Complutense de Madrid, Fac. Ciencias Políticas y Sociología, Dep. Relaciones Internacionales, 1989-1990.

106. González Pizarro, José Antonio: Politica de España en América bajo Isabel II. Univ. Navarra, Fac. Filosofía y Letras, 1985-1986.

107. Ho KuO SHIH: El proceso de integración centroamericana (1960-1993). Casaus Arzú, Marta Elena (Dir.). Univ. Complutense de Madrid, Fac. Geografía e Historia, Dep. Historia Contemporánea, 1995-1996.

108. IbÁÑEZ FERNÁNDEZ, Raquel Cecilia: Relaciones España-Uruguay, 1830-1900. Univ. Complutense de Madrid, Fac. Geografía e Historia, Dep. Historia de América I, 1989-1990.

109. LiAng Yung Ling: Continuidad y cambio en las relaciones entre la República Popular China y la ROC (Taiwan) con América Latina (1950-1995). Marquina Barrio, Antonio (Dir.). Univ. Complutense de Madrid, Fac. Ciencias Políticas y Sociología, Dep. Derecho Internacional y Relaciones Internacionales, 1996-1997.

110. Lluch Vélez, Amalia: La época de Muñoz Marín de contacto con el revolucionarismo mexicano-venezolano. Univ. Valladolid, Fac. Filosofía y Letras, Dep. Historia Moderna, Contemporánea y de América, 1989-1990.

111. LuBisco BRANCATO, Sandra María: Las relaciones hispano-brasileñas a fines del siglo XIX. Ballesteros Gaibrois, Manuel (Dir.). Univ. Complutense de Madrid, Fac. Geografía e Historia, 1983-1984.

112. Martínez Rivera, Agustín Alberto: Panamá y la construcción de un canal interoceánico en las relaciones exteriores de España en el siglo XIX (1845-1904). Univ. Complutense de Madrid, Fac. Geografía e Historia, 1989-1990.

113. Menezes Martín, Francisco Eduardo: Análisis comparativo de la prensa escrita española en el IV y V Centenario del Descubrimiento de América. Ventín Pereira, José Augusto (Dir.). Univ. Complutense de Madrid, Fac. Ciencias de la Información, Dep. Periodismo IV, 1995-1996.

114. NúÑEZ Polanco, Diomedes: Presencia norteamericana en el Caribe (Nicaragua y la República Dominicana, 1850-1870). Univ. Complutense de Madrid, Fac. Geografía e Historia, 1989-1990.

115. Ortiz Garza, José Luis: Valoración ético-jurídica de la actuación de las emisoras XER y XERA en la frontera de México y USA (1931-1941). Desantes Guanter, José María (Dir.). Univ. Navarra, Fac. Ciencias de la Información, Dep. Proyectos Periodísticos, 1994-1995.

116. Osorio MACHAdo, Lía: Mitos y realidades de la Amazonia brasileña en el contexto geopolítico mundial, 1540-1912. Univ. Barcelona, Fac. Geografía e Historia, 1988-1989. 
117. Pando Navarro, Concepción: La colonia española en México, 1930-1940. Nicolás Marín, María Encarna (Dir.). Univ. Murcia, Fac. Letras, Dep. Historia Moderna, Contemporánea y de América, 1993-1994.

118. PARdo SAnz, Rosa María: América Latina en la política exterior española, 1939-1945. Tusell Gómez, Javier (Dir.). Univ. Nal. Educación a Distancia, Fac. Geografía e Historia, Dep. Historia Contemporánea, 1992-1993.

119. QuiJAda MAURiÑo, Mónica Silvia: Relaciones hispano-argentinas: 1936-1948, coyunturas de crisis. Univ. Complutense de Madrid, Fac. Geografía e Historia, Dep. Historia de América, 1988-1989.

120. RuIZ JimÉnEZ, Laura: Estados Unidos y Gran Bretaña en la prensa diaria de Buenos Aires, 1926-1945. Malamud Riklés, Carlos (Dir.). Univ. Complutense de Madrid, Fac. Geografía e Historia, 1993-1994.

121. Sanahuja Perales, José Antonio: La ayuda norteamericana en Centroamérica, 1980-1992. Arenal Moyúa, Celestino del (Dir.). Univ. Complutense de Madrid, Fac. Ciencias Políticas y Sociología, Dep. Derecho Internacional Público y Relaciones Internacionales, 1995-1996.

122. SaPAg MuÑoz de la PeÑa, Pablo Vicente: Propaganda republicana y franquista en Chile durante la guerra civil española. Pizarroso Quintero, Alejandro (Dir.). Univ. Complutense de Madrid, Fac. Ciencias de la Información, Dep. Historia de la Comunicación Social, 1995-1996.

123. SCHMIED ZAPATA, Julie: Mecanismos de integración latinoamericana y el Estatuto Antártico: dos modelos de cooperación entre los países iberoamericanos. Ballesteros Gaibrois, Manuel (Dir.). Univ. Complutense de Madrid, Fac. Ciencias Políticas y Sociología, 1988-1989.

124. Sepúlveda MuÑoz, Isidro: Medio siglo de relaciones de España con las repúblicas americanas: El Movimiento Hispanoamericanista (1885-1936). Univ. Nal. Educación a Distancia, Fac. Geografía e Historia, Dep. Historia Contemporánea, 1991-1992.

125. Song, Guido: La política exterior mexicana ante la revolución cubana y nicaraguense. Calvo Buezas, Tomás (Dir.). Univ. Complutense de Madrid, Fac. Ciencias Políticas y Sociología, 1986-1987.

126. TABANERA GARCíA, Nuria: Las relaciones entre España e Hispanoamérica durante la Segunda República, 1931-1939: la acción diplomática republicana. González Loscertales, Vicente (Dir.). Univ. Valencia, Fac. Geografía e Historia, Dep. Historia Contemporánea, 1990-1991.

127. Vivas PinedA, Jesús Manuel: El arbitraje español en la cuestión de límites entre Venezuela y Colombia, 1881-1891. Univ. Complutense de Madrid, Fac. Geografía e Historia, Dep. Historia de América, 1986-1987.

Véase además: 142 y 150. 


\section{c) Historia Militar}

128. Solano Villanueva, Carmen Gabriela: La representación ofrecida por la prensa panameña a propósito de la institución militar (1968): Estudio de los relatos. Arias Fernández, M. Antonia (Dir.). Univ. Complutense de Madrid, Fac. Ciencias de la Información, Dep. Sociología IV, 1995-1996.

Véase además: 70, 80, 101 y 104.

d) Historia del Derecho

129. Сobo Carrasco, Mercedes: Análisis jurídico de "Doña Bárbara". Pérez-Prendes y Muñoz de Arraco, José Manuel (Dir.). Univ. Complutense de Madrid, Fac. Geografía e Historia, Dep. Historia Contemporánea, 1996-1997.

130. HARRIS FERnÁNDEZ, Jaime: Andrés Bello y el derecho del mar. Univ. Navarra, Fac. Derecho, 1980-1981.

131. ORdoñEs ChACón, Jaime: Una contribución al análisis de los derechos económicos, sociales y culturales en la evolución del estado de Costa Rica, 1948-1986. Univ. Complutense de Madrid, Fac. Derecho, Dep. Filosofía del Derecho, Moral y Política, 1989-1990.

132. Villamor Lucía, Fernando: La codificación penal en Bolivia. Rodríguez Devesa, José María (Dir.). Univ. Complutense de Madrid, Fac. Derecho, 1976-1977.

Véase además: 65 y 115.

e) Historia Económica

133. BARQUÍN LIAÑO, Julián: La ventaja comparativa y la política de protección en México: 1940-1980. Univ. Complutense de Madrid, Fac. Ciencias Económicas y Empresariales, 1985-1986.

134. BERETTA CURI, Alcides: El puerto de Barcelona y el comercio con América Latina (1898-1931): el programa americanista de la burguesía catalana. Univ. Barcelona, Fac. Geografía e Historia, 1984-1985.

135. Сно YоON Коок: La inserción de la economía argentina en la economía internacional a través del proceso de la gran inmigración europea (1870-1914). Univ. Complutense de Madrid, Fac. Ciencias Económicas y Empresariales, Dep. Economía Aplicada, 1987-1988.

136. DíAZ DE ARCiA, Amada Libertad: Política agraria en la República de Panamá. López Cepero Jurado, José Mariano (Dir.). Univ. Complutense de Madrid, Fac. Ciencias Políticas y Sociología, 1977-1978. 
137. Díaz Fuentes, Daniel: Crisis y cambios estructurales en América Latina: Un análisis comparado de la evolución económica y fiscal de Argentina, Brasil y México en el período de entreguerras. Comín Comín, Francisco (Dir.). Univ. Alcalá de Henares, Fac. Ciencias Económicas y Empresariales, Dep. Fundamentos de Economía e Historia Económica, 1991-1992.

138. Fernández García, José: Proceso de acumulación y crisis en México. Vidal Villa, José María (Dir.). Univ. Barcelona, Fac. Ciencias Económicas y Empresariales, Dep. Política Económica y Estructura Económica Mundial, 1994-1995.

139. FigueROA SÁNCHEZ, Isabel: El mercado mundial del cobre y el desarrollo chileno: 1950-1980. Varela Parache, Manuel (Dir.). Univ. Complutense de Madrid, Fac. Ciencias Económicas y Empresariales, Dep. Economía Aplicada I, 1992-1993.

140. GIRALDO IsAZA, Francisco Javier: El proceso de industrialización en Colombia, limitaciones y alcances. Univ. Barcelona, Fac. Ciencias Económicas y Empresariales, Dep. Política Económica, 1989-1990.

141. LóPEZ DEL AMO, Fernando: Ferrocarril, ideología y política ferroviaria en el proyecto liberal argentino (1852-1916). Univ. Barcelona, Fac. Geografía e Historia, 1988-1989.

142. LóPez SAlazAR, Pedro Eugenio: Los mecanismos de compensación en los procesos de integración económica entre países en desarrollo: el caso centroamericano (1960-1970). Alcántara Sáez, Manuel (Dir.). Univ. Complutense de Madrid, Fac. Geografía e Historia, Dep. Historia Contemporánea, 1995-1996.

143. Maroto BARQuero, Alberto: Aportación al estudio de la historia regional centroamericana: La estructura histórica de la provincia de Alajuela (Costa Rica), 1848-1963. Univ. Complutense de Madrid, Fac. Geografía e Historia, 1982-1983.

144. MARTíneZ DE SAN Vicente, Isabel: La construcción del territorio de las colonias de la "Central Argentine Land Company". Corominas Ayala, Miguel (Dir.). Univ. Politécnica de Cataluña, Esc. Tec. Sup. Arquitectura, Dep. Urbanismo y Ordenación del Territorio, 1994-1995.

145. Monteiro De CARvalho, Joao Carlos: O desenvolvimiento da agropecuaria brasileira da agricultura escravista ao sistema agroindustrial.[El desarrollo del sector agropecuario brasileño de la agricultura esclavista al sistema agroindustrial]. Voltes Bou, Pedro (Dir.). Univ. Barcelona, Fac. Ciencias Económicas y Empresariales, 1986-1987.

146. OYARZUN DE LAIGLESIA, Francisco Javier: Intercambio comercial de España con América en desarrollo durante el período 1961-85: un estudio de los flujos entre España y los países de la región desde ambas perspectivas. Univ. Complutense de Madrid, Fac. Ciencias Económicas y Empresariales, Dep. Economía Aplicada I, Economía Internacional y Desarrollo, 1988-1989. 
147. SAAVEDRA Restrepo, María Claudia: La conformación del mercado laboral urbano en los inicios del proceso de industrialización en Antioquia. Maluquer de Motes Bernet, Jordi (Dir.). Univ. Autónoma de Barcelona, Fac. Ciencias Económicas y Empresariales, Dep. Economía e Historia Económica, 1993-1994.

148. Santamaría García, Antonio: La industria azucarera y la economía cubana durante los años veinte y treinta. Bahamonde Magro, Angel (Dir.). Univ. Complutense de Madrid, Fac. Geografía e Historia, 1995-1996.

149. Segura Ivanchi, Antonio Enrique: Evolución bancaria Argentina, 1810-1980: análisis de políticas financieras. Bueno Campos, Eduardo (Dir.). Univ. Autónoma de Madrid, Fac. Ciencias Económicas y Empresariales, 1983-1984.

150. SiLVA, Benedito da: Las relaciones exteriores y el desarrollo de Brasil. Figueroa Martínez, Emilio (Dir.). Univ. Complutense de Madrid, Fac. Ciencias Económicas y Empresariales, 1980-1981.

Véase además: 42, 45, 60, 71, 73, 82, 90, 107, 121, 123, 154, 158, 169, 171 y 217.

\section{f) Historia Social, Etnohistoria y Demografía}

151. Abad GonZÁLEZ, Luisa: Etnocidio y procesos de cambio en la sociedad amazónica (caso aguaruna). Análisis del impacto de la cooperación al desarrollo con pueblos indios. Caravantes García, Carlos (Dir.). Univ. Complutense de Madrid, Fac. Geografía e Historia, Dep. Historia de América II, 1995-1996.

152. AKMIR CHAIB, Abdellahed: La emigración árabe a Argentina en la Historia contemporánea. Univ. Complutense de Madrid, Fac. Geografía e Historia, Dep. Historia de América, 1989-1990.

153. ANDREASSI CIERI, Alejandro: Movimiento huelguístico y socialismo en Argentina: Buenos Aires, 1895-1910. Martín Ramos, José Luis (Dir.). Univ. Autónoma de Barcelona, Fac. Filosofía i Lletres, 1994-1995.

154. Arbiol Quesada, Miguel: Empresariado monopólico: hegemonía social y evolución ideológica. Chile 1952-1983. Carreras Ares, Juan José (Dir.). Univ. Zaragoza, Fac. Filosofía y Letras, Dep. Historia Moderna y Contemporánea, 1996-1997.

155. AzCONA PASTOR, José Manuel: Los paraísos posibles. La emigración vasca a Argentina y Uruguay, 1830-1900. — [Bilbao: 1991, ISBN 84-7485-201-3]. García de Cortázar Ruiz de Aguirre, Fernando (Dir.). Univ. Deusto, 1990-1991. 
156. Bajo SAntos, Nicolás: Los dominicanos en Nueva York: Familia migrante y adaptación. Calvo Buezas, Tomás (Dir.). Univ. Complutense de Madrid, Fac. Ciencias Políticas y Sociología, Dep. Antropología Social, 1993-1994.

157. CABRera DÉnIZ, Gregorio José: Canarias y el regionalismo español en Cuba: de la Restauración a la Segunda República. Hernández García, Julio (Dir.). Univ. La Laguna, Fac. Geografía e Historia, Dep. Historia, 1991-1992.

158. Cagiao Vila, Pilar: Participación económico-social de los inmigrantes gallegos en Montevideo (1900-1970). Univ. Complutense de Madrid, Fac. Geografía e Historia, Dep. Historia de América I, 1989-1990.

159. CAÑEDO-ARgüElles FÁBREGA, Teresa: Relaciones interétnicas y proceso de formación social en la cuenca del Alto Paraná. Jiménez Núñez, Alfredo (Dir.). Univ. Sevilla, Fac. Geografía e Historia, Dep. Historia de América, 1984-1985.

160. Domínguez Prats, Pilar: Las exiliadas españolas en México: 1939-1950. Univ. Complutense de Madrid, Fac. Geografía e Historia, Dep. Historia Contemporánea, 1991-1992.

161. Escobar Belalcázar, Carlos Arnalfo: Las escogedoras de café en el Gran Caldos, 1930-40. Historia regional testimonial de su movimiento laboral sindical. Univ. Complutense de Madrid, Fac. Geografía e Historia, 1987-1988.

162. Fernández Esquivel, Francisco: Aportación al estudio del negro en Costa Rica. Participación política y discriminación racial. Hernández SánchezBarba, Mario (Dir.). Univ. Complutense de Madrid, Fac. Filosofía y Letras, 1976-1977.

163. Fernández Morales, María José: La emigración española a Venezuela de 1850 a 1960. Cuesta Domingo, Mariano (Dir.). Univ. Complutense de Madrid, Fac. Geografía e Historia, Dep. Historia de América, 1991-1992.

164. García García, Alejandro: Los Tarahumaras: historia de un etnocidio imposible. Univ. Murcia, Fac. Letras, Dep. Historia de América, 1984-1985.

165. Germani Guiomar, Inez: Políticas de asentamiento de población en el área rural de Brasil. Capel Sáez, Horacio (Dir.). Univ. Barcelona, Fac. Geografía e Historia, Dep. Geografía Humana, 1992-1993.

166. GIL ZúÑIGA, José Daniel: Homicidio, asociación y conflicto en la provincia de Heredia: 1895-1915. Fontana Lázaro, Josep (Dir.). Univ. Autónoma de Barcelona, Fac. Filosofía i Lletres, 1993-1994.

167. GonZÁlez MARIChal, Soledad: Familias campesinas mexicanas en el siglo XX. Gutiérrez Estévez, Manuel (Dir.). Univ. Complutense de Madrid, Fac. Geografía e Historia, Dep. Historia de América II, 1992-1993.

168. GonZÁlez MARTínez, Elda Evangelina: Españoles en Brasil: estudio acerca de la emigración española al Estado de Sao Paulo, 1880-1950. Univ. Complutense de Madrid, Fac. Geografía e Historia, 1988-1989. 
169. LoSADA ÁlVAREZ, Abel Fermín: Cambio demográfico y crecimiento económico en Cuba, 1898-1958. Las primeras fases de la transición demográfica. Dopico Gutiérrez del Arroyo, Fausto (Dir.). Univ. Santiago de Compostela, Fac. Ciencias Económicas y Empresariales, Dep. Historia e Instituciones Económicas, 1994-1995.

170. Montes Mozo, Segundo: Sociología del campesinado salvadoreño (el compadrazgo). Ballesteros Gaibrois, Manuel (Dir.). Univ. Complutense de Madrid, Fac. Filosofía y Letras, 1977-1978.

171. MoRey Russell MANYOKI, Rosenberg: Reforma agraria e incorporación de las poblaciones indígenas en Hispanoamérica (1917-1970). Hernández Sánchez-Barba, Mario (Dir.). Univ. Granada, Fac. Filosofía y Letras, 1982-1983.

172. Naranjo Orovio, Consuelo: La colonia española en Cuba, 1900-1959. Univ. Complutense de Madrid, Fac. Geografía e Historia, 1987-1988.

173. NorAmbuena CARrasco, Carmen Eliana: Las tendencias demográficas en la época republicana en Chile: El modelo de San Bernardo a través de los archivos parroquiales, 1824-1891. Hernández Sánchez-Barba, Mario (Dir.). Univ. Complutense de Madrid, Fac. Geografía e Historia, 1983-1984.

174. ORDEN, Liliana da: Redes sociales y espacios de interacción en los españoles de Mar del Plata (1895-1930). Llordén Miñambres, Moisés (Dir.). Univ. Oviedo, Fac. Geografía e Historia, Dep. Historia, 1995-1996.

175. Palau SuÁrez, Awilda: Veinticinco años del periódico independentista "Claridad" y su visión de 68 problemas sociales puertorriqueños. González Seara, Luis (Dir.). Univ. Complutense de Madrid, Fac. Ciencias Políticas y Sociología, 1985-1986.

176. PALAzÓn FerRANDo, Salvador: El movimiento migratorio español a Latinoamérica durante el último siglo (1882-1990). Gosálvez Pérez, Vicente (Dir.). Univ. Alicante, Fac. Filosofía y Letras, Dep. Geografía Humana, 1991-1992.

177. PÉREZ RUIZ, Roberto Aníbal: Historia social y antropológica de Cartagena (Chile). Univ. Complutense de Madrid, Fac. Geografía e Historia, Dep. Etnología y Antropología de América, 1984-1985.

178. PÉREZ TAYlOR y AldRETE, Rafael Antonio: Entre la tradición y la modernidad: Antropología de la memoria colectiva. Frigole Reisach, Joan (Dir.). Univ. Barcelona, Fac. Geografía e Historia, Dep. Antropología Social i Historia d'America i Africa, 1993-1994.

179. RodrígueZ OCAÑA, Horacio: Las migraciones interiores en la República de Panamá: 1800-1990. Puyol Antolín, Rafael (Dir.). Univ. Complutense de Madrid, Fac. Geografía e Historia, Dep. Geografía Humana, 1990-1991.

180. RuIZ DE LiRA Fuentes, Rafael: Problemas sociodemográficos de la América Central contemporánea a partir de un estudio de caso: Honduras. Puyol 
Antolín, Rafael (Dir.). Univ. Complutense de Madrid, Fac. Geografía e Historia, Dep. Historia Contemporánea, 1995-1996.

181. Salas Ramos, Carlos Manuel: El problema de la vivienda marginal en San José en el s. XX: Aportación a la Historia Social de Costa Rica. Hernández Sánchez-Barba, Mario (Dir.). Univ. Complutense de Madrid, Fac. Filosofía y Letras, Dep. Historia de América, 1977-1978.

182. SANTos, Ricardo Evaristo dos: La emigración española en la Bajada Santista - Estado de Sao Paulo - Brasil, 1880-1950. Univ. Complutense de Madrid, Fac. Geografía e Historia, 1988-1989.

183. SoldeVIlla ORIA, Consuelo: Emigración y exilio de los montañeses (1492-1975). La realidad del sueño americano. Rueda Hernanz, Germán (Dir.). Univ. Cantabria, Fac. Filosofía y Letras, Dep. Historia Moderna y Contemporánea, 1994-1995.

184. VÁzQuez Rial, Horacio: Las ideas sobre la población en el Río de la Plata en la época contemporánea. Capel Sáez, Horacio (Dir.). Univ. Barcelona, Fac. Geografía e Historia, Dep. Geografía Humana, 1990-1991.

185. Wiedmaier Burgos, Ana M.: Ideas y concepciones del pensamiento progresista chileno sobre la sociedad y las clases sociales en el siglo XIX y comienzos del siglo XX. Rubio Cordón, José Luis (Dir.). Univ. Complutense de Madrid, Fac. Ciencias Políticas y Sociología, Dep. Historia del Pensamiento y de los Movimientos Sociales y Políticos, 1990-1991.

186. YÁÑEZ GAllaRdo, César: Emigrar con red. La emigración catalana a América, 1830-1930. Nadal Oller, Jordi (Dir.). Univ. Autónoma de Barcelona, Fac. Lletres, Dep. Historia Moderna i Contemporania, 1993-1994.

Véase además: 56-59, 61, 64, 73-74, 77-78, 84-85, 90, 102, 117, 135, 145, $147,191,222$ y 247.

g) Historia de la Religión

187. Álvarez GILA, Oscar: La contribución europea a la Iglesia en el Río de la Plata: la presencia religiosa vasca (1835-1965). Escobedo Mansilla, Ronald Sixto (Dir.). Univ. País Vasco, Fac. Filología, Geografía e Historia, Dep. Historia Medieval, Moderna y de América, 1995-1996.

188. CoRTÉs MADRID, Antonio: La Iglesia y el Estado en Colombia en el siglo XIX. Univ. Complutense de Madrid, Fac. Geografía e Historia, 1981-1982.

189. Corvera PoIre, Marcela: Estudio histórico de la familia de franciscanos descalzos en la provincia de San Diego de México: siglos XVI-XX. Martínez de Codes, Rosa M. (Dir.). Univ. Complutense de Madrid, Fac. Geografía e Historia, Dep. Historia de América I, 1995-1996. 
190. FernÁndez FERnÁNDEZ, David: Historia de la Iglesia Católica en Santiago de Chile desde el Concilio Vaticano II hasta la actualidad: El testimonio oral como fuente. Pérez Murillo, M. Dolores (Dir.). Univ. Cádiz, Fac. Filosofía y Letras, Dep. Historia Moderna, Contemporánea, de América y del Arte, 1994-1995.

191. Gavilanes del Castillo, Luis María: Fe cristiana y cambio social en la diócesis de Riobamba: 1854-1894. Una aproximación al pensamiento social de Monseñor Proaño, Obispo de Indios. Univ. Complutense de Madrid, Fac. Ciencias Políticas y Sociología, 1985-1986.

192. Gelpi Barrios, Juan Rafael: La Iglesia y el Estado en Puerto Rico. Echeverría Martínez de Marigorta, Lamberto de (Dir.). Univ. Salamanca, Fac. Derecho, 1976-1977.

193. Gómez DíEz, Francisco Javier: El reformismo jesuítico en Guatemala en los años de la guerra fría (1945-1970). Hernández Sánchez-Barba, Mario (Dir.). Univ. Complutense de Madrid, Fac. Geografía e Historia, Dep. Historia de América I, 1993-1994.

194. Pazos Rodríguez, Antonio Manuel: La Iglesia en América en vísperas del concilio plenario latinoamericano de 1899. Estudio según la documentación vaticana. Andrés Gallego, José (Dir.). Univ. Navarra, Fac. Filosofía y Letras, Dep. Historia Contemporánea, 1991-1992.

195. PICCARDO, Diego R.: Estudio del concilio hispanoamericano convocado por León XIII en 1898. Pazos Rodríguez, Antón (Dir.). Univ. Navarra, Fac. Teología, 1990-1991.

196. Río SADORnIL, José Luis del: Labor misionera y cultural del padre Agustín López Pardo en la Amazonia peruana: la fundación de Requena. Univ. Complutense de Madrid, Fac. Geografía e Historia, 1990-1991.

197. Toro Jaramillo, Iván Darío: El clero en la diócesis de Medellín de 1868 a 1902. Actuación y formación sacerdotal. Pazos Rodríguez, Antón (Dir.). Univ. Navarra, Fac. Teología, Dep. Historia de la Iglesia, 1994-1995.

Véase además: 57, 65 y 199.

h) Historia Cultural y de la Educación

198. AdAMS FERnÁNDEZ, Carmen: "La Ilustración Española y Americana”: realidad y distorsión en la visión de América. Morales Saro, María Cruz (Dir.). Univ. Oviedo, Fac. Geografía e Historia, Dep. Historia del Arte y Musicología, 1996-1997.

199. Alonso Marañón, Pedro Manuel: Formación del profesorado y expansión educativa: Escuelas Pías en España y en América en el siglo XIX. Rodríguez Cruz, Agueda (Dir.). Univ. Salamanca, Fac. Ciencias de la Educación, Dep. Teoría e Historia de la Educación, 1995-1996. 
200. Barrantes Rodríguez, Manuel Antonio: El colegio de San Luis Gonzaga, una fundación española en Costa Rica, 1869-1910. Univ. Complutense de Madrid, Fac. Geografía e Historia, Dep. Historia de América, 1987-1988.

201. CAUTINO JoRdÁn, Eduardo: Ideas, actitudes políticas y producción historiográfica y literaria del grupo trascendentalista de Puerto Rico. Univ. Valladolid, Fac. Filosofía y Letras, 1986-1987.

202. Centeno de Navarro, Blanca Cecilia: Educación en el departamento de Tolima, Colombia, en el período comprendido entre 1886 y 1910. Univ. Complutense de Madrid, Fac. Filosofía y Ciencias de la Educación, 1989-1990.

203. Corral Sánchez-Cabezudo, Francisco: Vida y pensamiento de Rafael Barrett. Univ. Complutense de Madrid, Fac. Filosofía y Ciencias de la Educación, 1990-1991.

204. Delgado Imbert, M. del Pilar: El exilio cubano en Puerto Rico: su aportación cultural. Univ. Valladolid, Fac. Filosofía y Letras, Dep. Historia Moderna, Contemporánea y de América, 1989-1990.

205. Díaz Regadera, M. Dolores: José del Perojo y Figueras (1850-1908). Neokantismo y reformismo. Núñez Ruiz, Diego (Dir.). Univ. Autónoma de Madrid, Fac. Filosofía y Letras, Dep. Filosofía, 1995-1996.

206. Elvira QueZadA, Rocío: Análisis de la identidad cultural mexicana en el periodo 1940-1990. Marinas Herreras, José Miguel (Dir.). Univ. Complutense de Madrid, Fac. Ciencias Políticas y Sociología, Dep. Sociología IV, 1996-1997.

207. Fores Miravalles, Anna: Joan Bardina Castara, educador catalán, y sus proyecciones pedagógicas en Chile. Delgado Criado, Buenaventura (Dir.). Univ. Barcelona, Fac. Pedagogía, Dep. Teoría i Historia de l'Educació, 1996-1997.

208. García Morales, Alfonso: El Ateneo de México (1906-1914). Univ. Sevilla, Fac. Filología, Dep. Filologías Integradas, 1989-1990.

209. GonzÁlez RodríGUEZ, Dalma G.: Las aportaciones culturales de los exiliados españoles en Puerto Rico. Ramos Pérez, Demetrio (Dir.). Univ. Valladolid, Fac. Filosofía y Letras, Dep. Historia Moderna, Contemporánea y de América, 1996-1997.

210. GutiérRez Martínez, Delia Avelina: Ideología y educación en la República Dominicana, 1850-1903. Abellán García, José Luis (Dir.). Univ. Complutense de Madrid, Fac. Filosofía y Ciencias de la Educación, 1982-1983.

211. HERNÁNDEZ GARCÍA, Jesús: La educación en la obra narrativa y periodística de José Joaquín Fernández de Lizardi. Rodríguez Neira, Teófilo (Dir.). Univ. Oviedo, Fac. Ciencias de la Educación, 1996-1997.

212. HeRnándeZ PRIETO, María Isabel: Relaciones culturales entre Madrid e Hispanoamérica de 1881 a 1892. Sánchez-Castañer y Mena, Francisco (Dir.). Univ. Complutense de Madrid, Fac. Filología, 1979-1980. 
213. Herranz Herranz, Atanasio: Política del lenguaje en Honduras: 1502-1991. Alvar López, Manuel (Dir.). Univ. Complutense de Madrid, Fac. Filología, Dep. Filología Española I, 1993-1994.

214. KirSinger VÁSQuez, Liliana: Andrés Bello. Su proyección pedagógica. Univ. Barcelona, Fac. Pedagogía, Dep. Teoría e Historia de la Educación, 1989-1990.

215. JARQuín ORTEGa, María Teresa: Pensamiento educativo, ideología política e instituciones pedagógicas en el Estado de México. Epoca de la formación nacional. Hernández Sánchez-Barba, Mario (Dir.). Univ. Complutense de Madrid, Fac. Filosofía y Letras, 1976-1977.

216. JoRdÁn GARCÍA, Francisco Rafael: La planificación de la educación superior en Puerto Rico: 1960-1984. Univ. Complutense de Madrid, Fac. Filosofía y Ciencias de la Educación, 1989-1990.

217. LÁrez Romero, Ronald José: Dependencia económica e identidad cultural de América Latina: Cuba, Nicaragua y Venezuela. Univ. Barcelona, Fac. Ciencias Económicas y Empresariales, 1987-1988.

218. Ledesma ReYes, Manuel: Krausismo y educación en Costa Rica. La influencia de los educadores canarios: Valeriano y Juan Fernández Ferraz. Univ. La Laguna, Fac. Filosofía y Ciencias de la Educación, Dep. Historia y Filosofía de la Ciencia, la Educación y el Lenguaje, 1994-1995.

219. MARTíNEZ DE CODES, Rosa María: El método generacional: su aplicación a la historia del pensamiento argentino. Peset Reig, José Luis (Dir.). Univ. Complutense de Madrid, Fac. Geografía e Historia, 1984-1985.

220. Molina GuZmán, Guillermo: Evolución de la educación costarricense desde la colonia hasta 1980. Su crisis actual. Univ. Barcelona, Fac. Filosofía y Ciencias de la Educación, 1985-1986.

221. Montero Segura, Isaac: Las proyecciones históricas del krausismo español en Costa Rica, 1870-1936. Martínez Quintero, M. Esther (Dir.). Univ. Complutense de Madrid, Fac. Geografía e Historia, Dep. Historia de América I, 1994-1995.

222. MulleR, Verónica Regina: El niño ciudadano y otros niños. Concepciones de infancia en una perspectiva histórica y sus relaciones con el "niño" del Ayuntamiento de Porto Alegre. Núñez Pérez, Violeta (Dir.). Univ. Barcelona, Fac. Pedagogía, Dep. Teoría e Historia de la Educación, 1996-1997.

223. Nieto Sotelo, Jesús: La Universidad Nacional de México durante la Revolución mexicana (1910-1921). Peset Reig, Mariano (Dir.). Univ. Valencia, Fac. Geografía e Historia, Dep. Historia Contemporánea, 1993-1994.

224. Ossenbach SAUTER, Gabriela: Formación de los sistemas educativos nacionales en Hispanoamérica: la política educativa como factor de consolidación del Estado Nacional (1870-1900). El caso del Ecuador. Univ. Nal. Educación a Distancia, Fac. Filosofía y Ciencias de la Educación, 1987-1988. 
TESIS ESPAÑOLAS SOBRE HISTORIA DE AMÉRICA LATINA (1976/77-1996/97)

225. Patiño Rodríguez, Enilda: Historia de la educación normal panameña. Gutiérrez Zuluaga, Isabel (Dir.). Univ. Complutense de Madrid, Fac. Filosofía y Ciencias de la Educación, 1982-1983.

226. Perea EnRíQuez, Héctor Manuel: Presencia de México y de los intelectuales mexicanos en la prensa española (1914-1936). Portal Nicolás, Marta (Dir.). Univ. Complutense de Madrid, Fac. Ciencias de la Información, Dep. Filología Española III, 1994-1995.

227. Pereira Domínguez, María del Carmen: La labor educativa iberoamericana y la creación de las Escuelas Pro-Valle Miñor a principios del siglo XX. Univ. Complutense de Madrid, Fac. Filosofía y Ciencias de la Educación, Dep. Teoría e Historia de la Educación, 1987-1988.

228. PÉREZ QuiÑones, Luz Ivette: Trayectoria de la instrucción en el Puerto Rico del siglo XIX y la educación superior ante el cambio de soberanía. Mijares Pérez, Lucio (Dir.). Univ. Valladolid, Fac. Filosofía y Letras, Dep. Historia Moderna, Contemporánea y de América, 1994-1995.

229. ReIMUNDE NoReÑA, Dolores: Hispanoamérica en las revistas gallegas (1914-1936). Univ. Complutense de Madrid, Fac. Filología, 1988-1989.

230. RodríGUEZ GuILlÉn, Rafael: Eugenio María de Hostos, reformador escolar en Hispanoamérica. Ruiz Berrio, Julio (Dir.). Univ. Complutense de Madrid, Fac. Filosofía y Ciencias de la Educación, 1979-1980.

231. SÁnchez VAlLe, Ignacio: Análisis documental de la pedagogía general a través de los textos de autores hispanoamericanos (1932-1982). Univ. Complutense de Madrid, Fac. Filosofía y Ciencias de la Educación, 1985-1986.

232. Sandi Murillo, Manuel Alberto: La educación de adultos en Costa Rica. Romero Marín, Anselmo (Dir.). Univ. Complutense de Madrid, Fac. Filosofía y Letras, 1977-1978.

233. Santa María Rodríguez, Carlos: Poder y educación en Chile, periodos: Frei, Allende, Pinochet. Univ. Barcelona, Fac. Filosofía y Ciencias de la Educación, Dep. Pedagogía Sistemática, 1984-1985.

Véase además: 56, 72, 90, 92, 97, 113, 115, 120 y 124.

\section{i) Historia de la Ciencia}

234. CASAls CoSTA, Vicente: La formación científica, la actividad espacial y la proyección intelectual de los ingenieros de montes en la España contemporánea (1848-1936). Capel Sáez, Horacio (Dir.). Univ. Barcelona, Fac. Geografía e Historia, Dep. Geografía Humana, 1994-1995.

235. Estarlich CAnet, José Vicente: Emilio Mira y López: su vida y su obra científica. Rey González, Antonio (Dir.). Univ. Valencia, Fac. Medicina y Odontología, 1992-1993. 
236. Gangas GeIsSE, Mónica: La evolución de la Geografía chilena durante el siglo XX: contextos, tendencias y autores. Univ. Barcelona, Fac. Geografía e Historia, 1984-1985.

237. LÓPEZ-OCÓN CABRERA, Leoncio: De viajero naturalista a historiador: las actividades americanistas del científico español Marcos Jiménez de la Espada (1831-1898). Univ. Complutense de Madrid, Fac. Geografía e Historia, 1989-1990.

238. Mendoza Vargas, Héctor: Ciencia, Estado y burocracia en el México independiente: La biografía científica del ingeniero geógrafo Francisco Díaz Covarrubias (1833-1889). Capel Sáez, Horacio (Dir.). Univ. Barcelona, Fac. Geografía e Historia, Dep. Geografía Humana, 1996-1997.

239. Puig-Samper Mulero, Miguel Angel: La Comisión Científica del Pacífico (1862-1866). Univ. Complutense de Madrid, Fac. Biología, 1986.

240. Río-Hortega Bereciartu, Juan del: El epistolario de D. Pío del RíoHortega. Riera Palmero, Juan (Dir.). Univ. Valladolid, Fac. Medicina y Cirugía, Dep. Medicina, 1991-1992.

241. Torralba Martínez, Antonio: Antonio Machado y Núñez (1815-1896) y su contribución a la cultura española. Orozco Acuaviva, Antonio (Dir.). Univ. Cádiz, Fac. Medicina, Dep. Anatomía Patológica, Biología Celular e Historia de la Ciencia, 1993-1994.

\section{j) Urbanismo}

242. Álvarez Prozorovich, Fernando Victorino: El sueño moderno en Buenos Aires (1930-1949). Univ. Politécnica de Cataluña, Esc. Tec. Sup. Arquitectura, 1991-1992.

243. ERNST FoRTUNATTI, Raymundo Javier: Del Santiago colonial a la capital republicana. Bonet Correa, Antonio (Dir.). Univ. Salamanca, Fac. Geografía e Historia, Dep. Historia del Arte, 1995-1996.

244. Felsenhardt Rosen, Silvia Cristina: El impacto de los cambios sociopolíticos en la arquitectura de vivienda. Caso Chile, 1958-1989. Bohigas Guardiola, Oriol (Dir.). Univ. Politécnica de Cataluña, Esc. Tec. Sup. Arquitectura, Dep. Composición Arquitéctonica, 1993-1994.

245. Ferreira Martins, Carlos Alberto: Razón, ciudad y naturaleza. González Amezqueta, Adolfo (Dir.). Univ. Politécnica de Madrid, Esc. Tec. Sup. Arquitectura, Dep. Composición Arquitectónica, 1991-1992.

246. Garcés Feliú, Eugenio: Las ciudades del salitre: un estudio comparativo de los modelos de asentamiento salitreros en la región de Antofagasta (Chile). El caso de las oficinas María Elena y Pedro de Valdivia. Univ. Politécnica de Cataluña, Esc. Tec. Sup. Arquitectura, 1986-1987. 
TESIS ESPAÑOLAS SOBRE HISTORIA DE AMÉRICA LATINA (1976/77-1996/97)

247. Loures Seone, M. Luisa: Buenos Aires: centro histórico y crisis social. Urrutia Abaigar, Víctor (Dir.). Univ. Complutense de Madrid, Fac. Ciencias Políticas y Sociología, Dep. Sociología I, 1996-1997.

248. SÁnchez Martínez, Manuel: El sector Reforma de Guadalajara. Un espacio social planificado para el riesgo. Alvarez Mora, Alfonso (Dir.). Univ. Valladolid, Fac. Arquitectura, Dep. Análisis e Instrumentos de Intervención Arquitectónica y Urbanística, 1996-1997.

Véase además: 181 y 196.

k) Historia del Arte

249. Cabañas Bravo, José Miguel: La Primera Bienal Hispanoamericana de Arte: Arte, política y polémica en un certamen internacional de los años cincuenta. Arias Anglés, Enrique (Dir.). Univ. Complutense de Madrid, Fac. Geografía e Historia, Dep. Historia del Arte, 1990-1991.

250. Cazalla Piñero, Juana M.: Arte cubano de fin de siglo. Martínez Muñoz, Amalia (Dir.). Univ. Politécnica de Valencia, Fac. Bellas Artes, Dep. Historia del Arte, 1994-1995.

251. Corella Lacasa, Miguel: El estridentismo y las Artes. Aproximación a la vanguardia mexicana en la década de los veinte. Tormo Fayos, José Enrique (Dir.). Univ. Politécnica de Valencia, Fac. Bellas Artes, Dep. Historia del Arte, 1996-1997.

252. Fernández García, Ana M.: Pintura española en Buenos Aires, 1880-1930. Morales Saro, María Cruz (Dir.). Univ. Oviedo, Fac. Geografía e Historia, Dep. Historia y Artes, 1992-1993.

253. FORMENT ROMERO, Albert: Vanguardia artística y compromiso político: vida y obra de Josep Renau. Aguilar Civera, Inmaculada (Dir.). Univ. Valencia, Fac. Geografía e Historia, Dep. Historia del Arte, 1994-1995.

254. GonzÁlez Lamela, M. Pilar: La guerra civil española y el exilio artístico. Urrea Fernández, Jesús (Dir.). Univ. Valladolid, Fac. Filosofía y Letras, Dep. Historia del Arte, 1994-1995.

255. Guerrero Rodríguez, Aura Coromoto: Génesis y evolución de la pintura de paisaje en Venezuela (1790-1912). Pena López, Carmen (Dir.). Univ. Complutense de Madrid, Fac. Geografía e Historia, Dep. Historia del Arte III, 1994-1995.

256. GutiÉRreZ ViñuALes, Rodrigo: La pintura en Argentina (1880-1930). En busca de una identidad nacional. López Guzmán, Rafael (Dir.). Univ. Granada, Fac. Filosofía y Letras, Dep. Historia del Arte, 1995-1996.

257. PÉrez Lizano Forns, Manuel: Arte contemporáneo de Puerto Rico, 1950-1983. Fotografía y grabado. Univ. Zaragoza, Fac. Filosofía y Letras, Dep. Historia del Arte, 1987-1988. 
258. SalCedo Miliani, Antonio: Armando Reverón y su época. Univ. Barcelona, Fac. Geografía e Historia, 1986-1987.

259. Tío FeRnÁNDEZ, Teresa: Origen y desarrollo de la escuela puertorriqueña del cartel. Urrea Fernández, Jesús (Dir.). Univ. Valladolid, Fac. Filosofía y Letras, Dep. Historia del Arte, 1992-1993.

260. VIEIRA, Silvia Valeria: Oswald de Andrade: el antropófago y el hombre cordial. Subirats Ruggeberg, Eduardo (Dir.). Univ. Nal. Educación a Distancia, Fac. Filosofía, 1995-1996.

261. WeChSLER, Diana Beatriz: Crítica de arte, condicionadora del gusto. La consagración y el consumo de obras de arte. Buenos Aires, 1920-1930. Henares Cuellar, Ignacio (Dir.). Univ. Granada, Fac. Filosofía y Letras, Dep. Historia del Arte, 1994-1995.

262. Zamorano Pérez, Pedro Emilio: Fernando Alvárez de Sotomayor y la generación del centenario: influencia española en la pintura chilena. Univ. Complutense de Madrid, Fac. Geografía e Historia, 1986-1987. 